\title{
First do no harm - A case of limb shaking TIA
}

Sir,

A 75-year-old gentleman presented to us with the chief complaint of weakness of right lower limb. The same morning, patient complained of shaking of both lower limbs approximately in the frequency of 3-4 Hz and particularly the symptoms were present when he stood. The shaking stopped as he lied down. There was no marching of the involuntary movements. He was on medications for diabetes mellitus and hypertension. He was assessed elsewhere in the morning and the involuntary movements were attributed to seizure and was advised EEG which was normal. His blood pressure (BP) was 220/110 $\mathrm{mmHg}$ and he was given $10 \mathrm{mg}$ of nimodipine by the family physician. He was started on oral phenytoin. When he presented to us, he had difficulty in lifting the right lower limb. He was dragging the right lower limb while walking. His neurological examination revealed right crural monoplegia with motor power of $3 / 5$. Rest of the neurological examinations was normal. Auscultation of carotids revealed left carotid bruit. His BP was $120 / 90 \mathrm{mmHg}$.

Magnetic resonance angiography (MRA) of the brain revealed watershed infarcts in both cerebral hemispheres in the anterior cerebral artery and middle cerebral artery (ACA-MCA) regions, and complete occlusion of right internal carotid artery (ICA) and severe stenosis of left carotid artery [Figures 1 and 2]. His carotid duplex showed complete absence of flow in the right carotid artery and left carotid artery showed $60-70 \%$ stenosis.

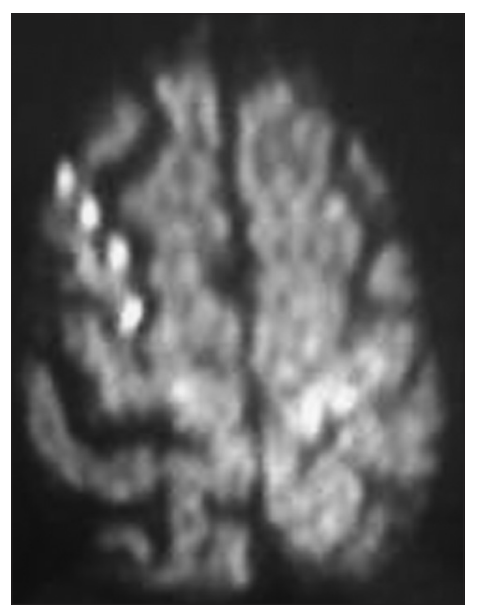

Figure 1: Diffusion weighted image showing bilateral cortical watershed infarcts 


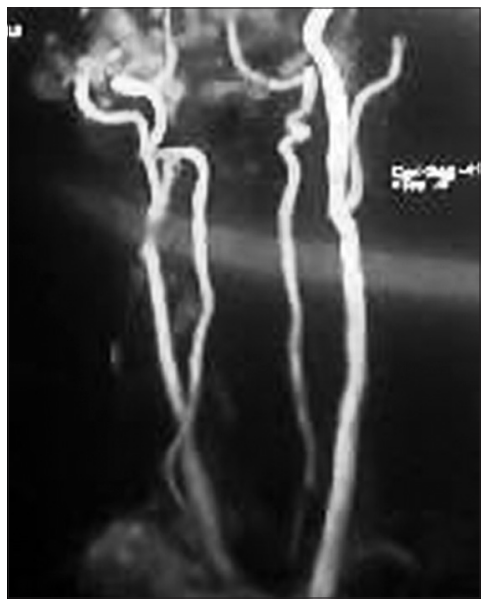

Figure 2: Magnetic resonance angiography neck vessels showing complete occlusion of right internal carotid artery and severe stenosis of left carotid artery

The patient was treated conservatively with antiplatelets as he denied cerebral angiography for possible reperfusion intervention. At times, keeping the blood pressure on higher side can benefit such patients from further progression, but with the background of atherosclerotic diseases like diabetes and hypertension, it poses a great risk to myocardium and kidneys.

Miller Fisher first described a temporary limb shaking syndrome (LSS) associated with carotid stenosis in 1962; since then, the diagnosis has been reported regularly though only 48 cases have been recorded so far as per our review and rather than being rare, it seems to be a misrecognized syndrome. ${ }^{[1]}$ However bilateral shaking of lower extremities is a very rare presentation. ${ }^{[2]}$ The clinical features of LSS comprise a group of rhythmic or arrhythmic involuntary hyperkinesias affecting the hand, arm, leg, hand-arm, or hand-arm-leg unilaterally. They can be very easily be mistaken for focal seizures though there is no jacksonian march and there is no involvement of the face, as what happened in our patient. Patients describe these movements as shaking, jerking, twitching, or trembling. Associated symptoms may include ataxia, myoclonus, dystonic limb posturing, and Parkinsonism. ${ }^{[3]}$ These symptoms are often brought out by postural change and at times relieved by sitting or lying down.

The exact mechanism of LSS is unclear, though it can be postulated to be due to the 'hypoperfusion' theory in which the carotid stenosis leads to decreased blood supply to critical watershed territories in the brain. This is further supported by the fact that these movements are provoked by maneuvers that cause cerebral hypoperfusion. A lot of studies have shown the hemodynamic failure as the underlying cause of the LSS. EEGs have failed to show epileptiform activity, although some patients do show contralateral slowing. ${ }^{[4]}$ The most striking feature of LSS is severe internal carotid artery stenosis.
In patients with stenosis of carotid arteries, carotid endarterectomy is the procedure of treatment of choice. ${ }^{[5]}$ However with the improved technology of stents and distal protection devices, endovascular treatment offers a favorable role in select cases. ${ }^{[6]}$ Acute carotid occlusions have been stented following angioplasty showing favorable results but further studies are required to make it a standard practice. Thus, identification of LSS and detection of underlying carotid artery disease is crucial for prevention of stroke.

\section{Sweta P. Adatia, Vinay S. Chauhan, Shirish M. Hastak}

Department of Neurology, Lilavati Hospital and Research Center, Bandra (West), Mumbai - 400 050, India. E-mail: sweta.adatia@gmail.com

\section{References}

DOI: $10.4103 / 0028-3886.59486$

1. Fisher CM. Concerning recurrent transient cerebral ischemic attacks. Can Med 1962;86:1091-9.

2. Galvez-Jamenez, Hanson MR, Hangreave MJ, Peirut P. Transient ischemic attacks and paroxysmal dyskinesia: An under-recognized association. Adv Neurol 2002;89:421-32.

3. Kowacz PA, Troiano AR, Mendonca CT, Teive HA, Werneck LC. Caraotid transient ischemic attacks presenting as limb-shaking syndrome. Arq Neuropsiquiatr 2004;62:339-41.

4. Ali S, Khan MA, Khealani B. Limb-shaking transient ischemic attacks: Case report and review of literature. BMC Neurol 2006.

5. Tatemichi TK, Young WL, Prohovnik I, Gitelman DR, Correll JW, Mohr JP. Perfusion insufficiency in limb-shaking transient ischemic attacks. Stroke 1990;21:341-7.

6. Yadav JS, Wholey MH, Kuntz RE, Fayad P, Katzen BT, Mishkel GJ, et al. Protected carotid-artery stenting versus endarterectomy in high risk patients. N Engl J Med 2004;351:1493-501.

Accepted on 09-03-2009 